\title{
Chemopreventive Effects of Sarcotriol on Ultraviolet B-induced Skin Tumor Development in SKH-1 Hairless Mice
}

\author{
Vipra Kundoor ${ }^{1}$, Xiaoying Zhang ${ }^{1}$, Ajay Bommareddy ${ }^{1}$, Sherief Khalifa ${ }^{2}$, Hesham Fahmy ${ }^{1, *}$ \\ and Chandradhar Dwivedi ${ }^{1}$ \\ ${ }^{1}$ Department of Pharmaceutical Sciences, South Dakota State University, Box 2202C, Brookings, SD \\ 57007, USA. Tel.: 605-688-4243, Fax 605-688-5993. \\ 2 Department of Pharmacognosy, Faculty of Pharmacy, Suez Canal University, Ismailia, Egypt \\ * Author to whom correspondence should be addressed; E-mail: Hesham.Fahmy@sdstate.edu
}

Received: 2 October 2007 / Accepted: 11 December 2007 / Published: 12 December 2007

\begin{abstract}
Sarcotriol (ST) has been shown to be chemopreventive on 7,12-dimethylbenz(a)anthracene (DMBA) initiated and 12-O-tetradecanoylphorbol-13-acetate (TPA)promoted skin tumor development in CD-1 mice in recent studies from our laboratory. The objective of this study was to determine the chemopreventive effects of ST on ultraviolet B (UVB)-induced skin tumor development in female SKH-1 hairless mice, an experimental model relevant to human skin cancer development, and its possible mechanisms of action. Female SKH-1 mice were divided into two groups: Control and ST treated. Control was topically treated with $100 \mu \mathrm{L}$ acetone and ST treated group administered with $30 \mu \mathrm{g}$ ST in $100 \mu \mathrm{L}$ acetone one hour before UVB exposure. For UVB-induced tumorigenesis, carcinogenesis was initiated and promoted by UVB $\left(180 \mathrm{~mJ} / \mathrm{cm}^{2}\right)$. Group weights and tumor counts were taken once every week. After 30 weeks, mice were sacrificed and dorsal skin samples were collected. The proteins from the skin sample were further used for SDSPAGE and Western blotting using specific antibodies against caspase-3, caspase-8, caspase- 9 and p53. Tumor multiplicity was found 19.6, 5.2 in the control and ST treated groups respectively. Caspase-3, $-8,-9$ and p53 were significantly $(P<0.05)$ upregulated in ST treated group compared to Control group. Together, this study for the first time identifies the chemopreventive effects of ST in UVB-induced carcinogenesis possibly by inducing apoptosis and upregulating p53.
\end{abstract}


Keywords: Cancer chemoprevention, Skin cancer, Cembranoids, Sarcophine, Sarcophyton glaucum, Sarcotriol, Apoptosis, p53

\section{Introduction}

Skin cancer is the most common of all cancer types, leading to the diagnosis of more than 1 million cases each year in the United States. An estimated 10,850 deaths will occur in 2007 [1], resulting from repeated sunlight exposure in which ultraviolet B (UVB) radiation is a major environmental carcinogen that induces nonmelanoma skin cancer [2-4].

Chemical and UVB radiation-induced carcinogenesis in murine and humans is a multistep process and has been divided into defined stages of initiation, promotion and progression [5]. UVB acts both as an initiator as well as promoter. UVB radiation is absorbed by DNA which causes damage to the DNA, primarily at sites of adjacent pyrimidines in the form of dimers [3, 4, 6]. The generation of DNA photoproducts has been shown to initiate tumorigenesis. Mechanism(s) of the UVB-induced promotion are not been fully understood. Several factors such as generation of reactive oxygen species causing oxidative stress and activation of various signaling cascades including the synthesis of prostaglandins (PG) play a major role in clonal expansion of UVB-initiated cells into visible skin tumors [7-9].

Developmrnt of novel strategies to prevent skin cancer represents a desirable goal due to the increasing rise in incidence of skin cancer patients throughout the world [10,11]. Chemoprevention is a means of cancer control in which the occurrence of the disease can be entirely prevented, slowed down or reversed by the administration of one or more naturally occurring and/or synthetic compounds $[12,13]$. Nowadays, the supplementation or topical application of synthetic agents viz. retinoids, vitamins, inhibitors of ornithine decarboxylase, cyclooxygenase, lipooxygenase and other antioxidant compounds including thiols and minerals have gained much attention on one hand, while the use of natural agents like polyphenols, monoterpenes, flavonoids, organosulphides, indoles etc have shown promise for their development as chemopreventive agents against skin cancer [14, 15].

Recent studies have focused on the potential applications of marine organisms, particularly in the treatment of human diseases [16]. Several marine natural products are in pre-clinical and clinical evaluation, one of which is sarcophytol A [17]. This is a hydroxylated cembranoid isolated from the soft coral Sarcophyton glaucum that has shown cancer chemopreventive activity [18]. Another related cembranoid is sarcophine, obtained from the Red Sea soft coral Sarcophyton glaucum, which was found to possess diverse biological activity [19]. Sarcotriol (ST), a semi-synthetic derivative of sarcophine has shown higher activity than the known chemopreventive agent sarcophytol A [18] The structures of sarcophine and sarcotriol are shown in Figure 1. Recent studies from our laboratory have shown significant chemopreventive effects of ST on 7,12-dimethylbenz(a)anthracene (DMBA)initiated and 12-O-tetradecanoylphorbol-13-acetate (TPA)-promoted skin tumor development in female CD-1 mice by inducing apoptosis and decreasing COX-2 levels [20]. These promising results prompted this investigation to study the effects of ST on UVB-induced tumorigenesis in female SKH-1 hairless mice model. 
Figure 1. Structures of sarcophine and sarcotriol.

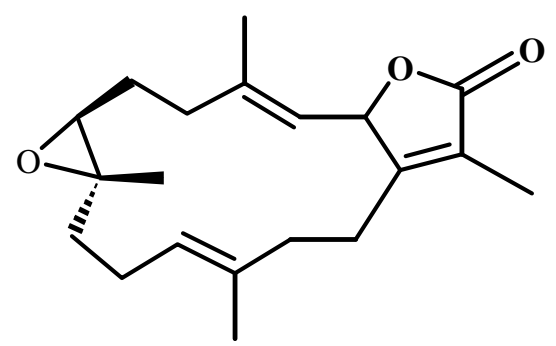

Sarcophine

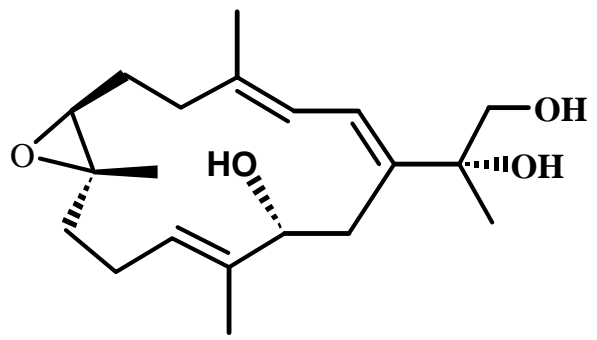

Sarcotriol

The objective of this study was to determine the chemopreventive effects of ST on UVB-induced skin tumor development in female SKH-1 hairless mice, an experimental model relevant to human skin cancer development. One of the biological responses for tumorigenesis is the loss of apoptotic death. Caspase-3, -8 and -9 are proapoptotic proteins which could induce apoptosis, whereas, p53, the tumor suppressor gene is involved in many cellular functions including cell cycle inhibition, regulation of differentiation, transcription, DNA repair, and apoptosis of cells sustaining DNA damage [3,4]. Therefore, the effects of ST on the expressions of caspase-3, -8, -9 and p53 after UVB exposure to SKH-1 mice also were determined to elucidate the possible mechanisms of action of ST.

\section{Results and Discussion}

The effects of ST treatment one hour before the UVB exposure on the tumor incidence and multiplicity are shown in Figures 2a and 2b, respectively.

Figure 2a. The effects of ST pretreatment on tumor incidence in UVB-induced tumorigenesis in female SKH-1 hairless mice. ST pretreatment delayed the onset of tumors from $12^{\text {th }}$ week till $15^{\text {th }}$ week when compared to the control group.

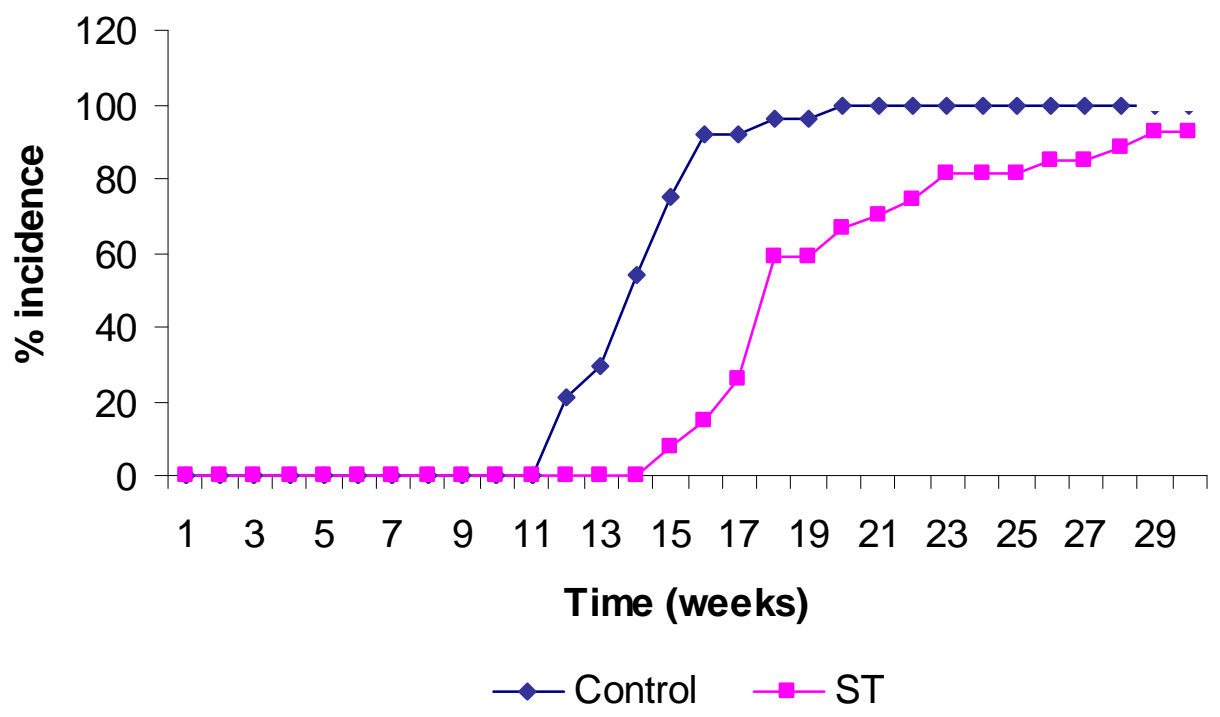


Figure 2b: The effects of ST pretreatment on tumor multiplicity in the UVB-induced tumorigenesis in female SKH-1 hairless mice. ST decreased tumor multiplicity throughout the experiment. Each point represents mean number of tumors per mice $\pm \mathrm{SE}$ derived from 27 mice.

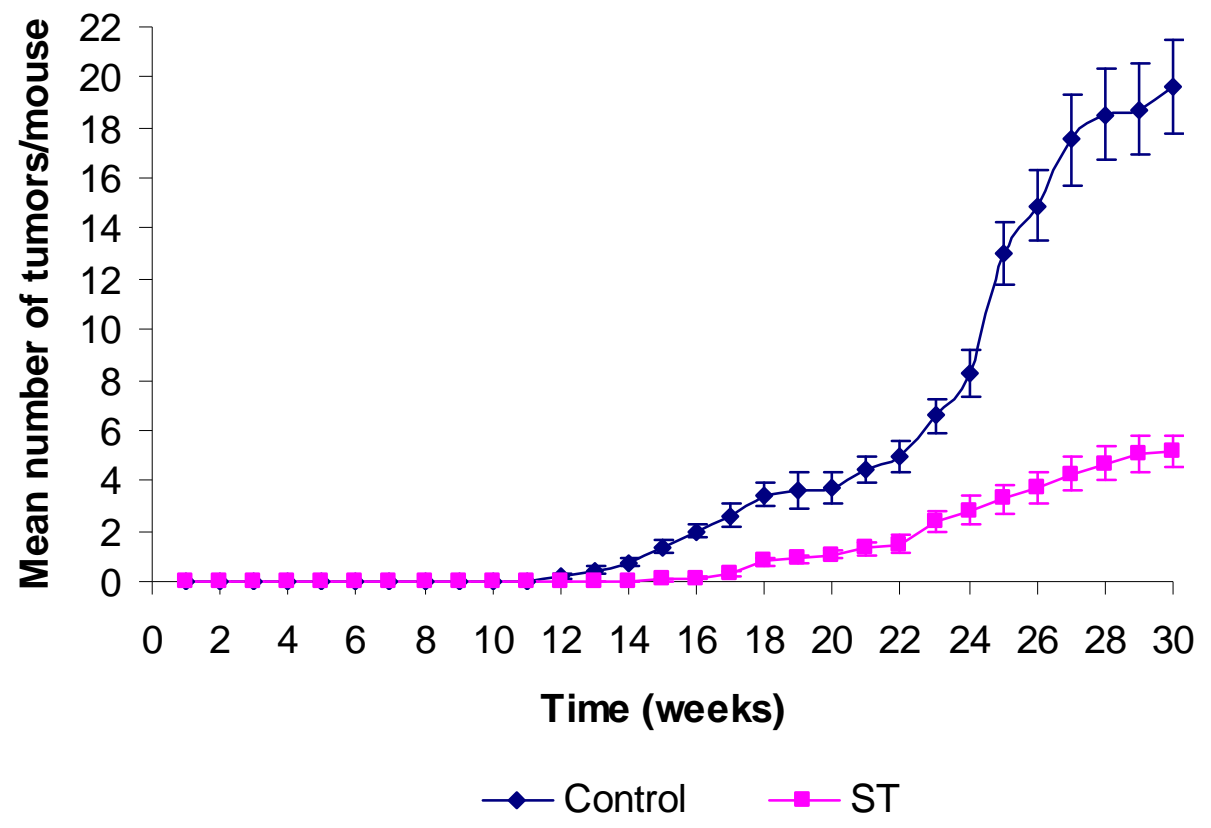

The results of the present investigation revealed a delay in the onset of tumorigenesis in the animals pretreated with ST. In the Control group, the onset of tumors commenced at $12^{\text {th }}$ week of promotion and reached $100 \%$ by the $20^{\text {th }}$ week, whereas pretreatment with ST one hour before UVB, the onset of tumors were delayed until the $15^{\text {th }}$ week of promotion and, moreover, it was only $75 \%$ until the $23^{\text {rd }}$ week. Tumor incidence was significantly $(\mathrm{p}<0.05)$ lower in ST treated group than Control group until $28^{\text {th }}$ weeks of promotion. By the end of the experiment, tumor incidence was found to be 100 and $92 \%$ in the Control and ST treated group respectively. As shown in Figure 2b, ST treatment resulted in a significant $(P<0.05)$ reduction in the number of tumors per mouse compared to the Control group throughout the duration of experiment. The mean number of tumors at the end of experiment in Control and ST treated group was found to be 19.6 and 5.2 respectively. These results indicated that ST inhibited skin tumor development in both tumor incidence, to a lesser extent, and tumor multiplicity in the ST treated groups (Figure 3). There was no significant difference in the average mouse weight between the Control and ST treated group throughout the experiment (data not shown). Moreover, the skin of mice that were treated with ST appeared to be healthy and no toxic symptoms were observed.

One biological event responsible for tumorigenesis is the loss of apoptotic death of transformed cells [21]. In most cancers, the apoptotic pathway of tumor cells is compromised with a survival advantage over the normal cells [22]. Induction of apoptosis is a key event, which controls the outcome of chemopreventive efficacy of an agent under investigation [22, 23]. A fundamental biochemical event that starts apoptosis is the activation of caspases [24]. Apoptosis occurs through two pathways; extrinsic and intrinsic [25]. The extrinsic pathway is activated by the ligation of death receptors, which ultimately leads to the activation of caspase-8 [25]. In the intrinsic pathway of 
apoptosis, mitochondrial dysfunction takes place causing the release of cytochrome $c$ into the cytosol, which finally leads to the activation of caspase-9 [26]. Finally the upstream caspases-9 and -8 converge to caspase-3 leading to apoptosis [25].

Figure 3: The skin cancer protective effect of sarcotriol: skin tumor incidence and multiplicity were significantly reduced in mice pretreated with sarcotriol (bottom) compared to the untreated control (top).

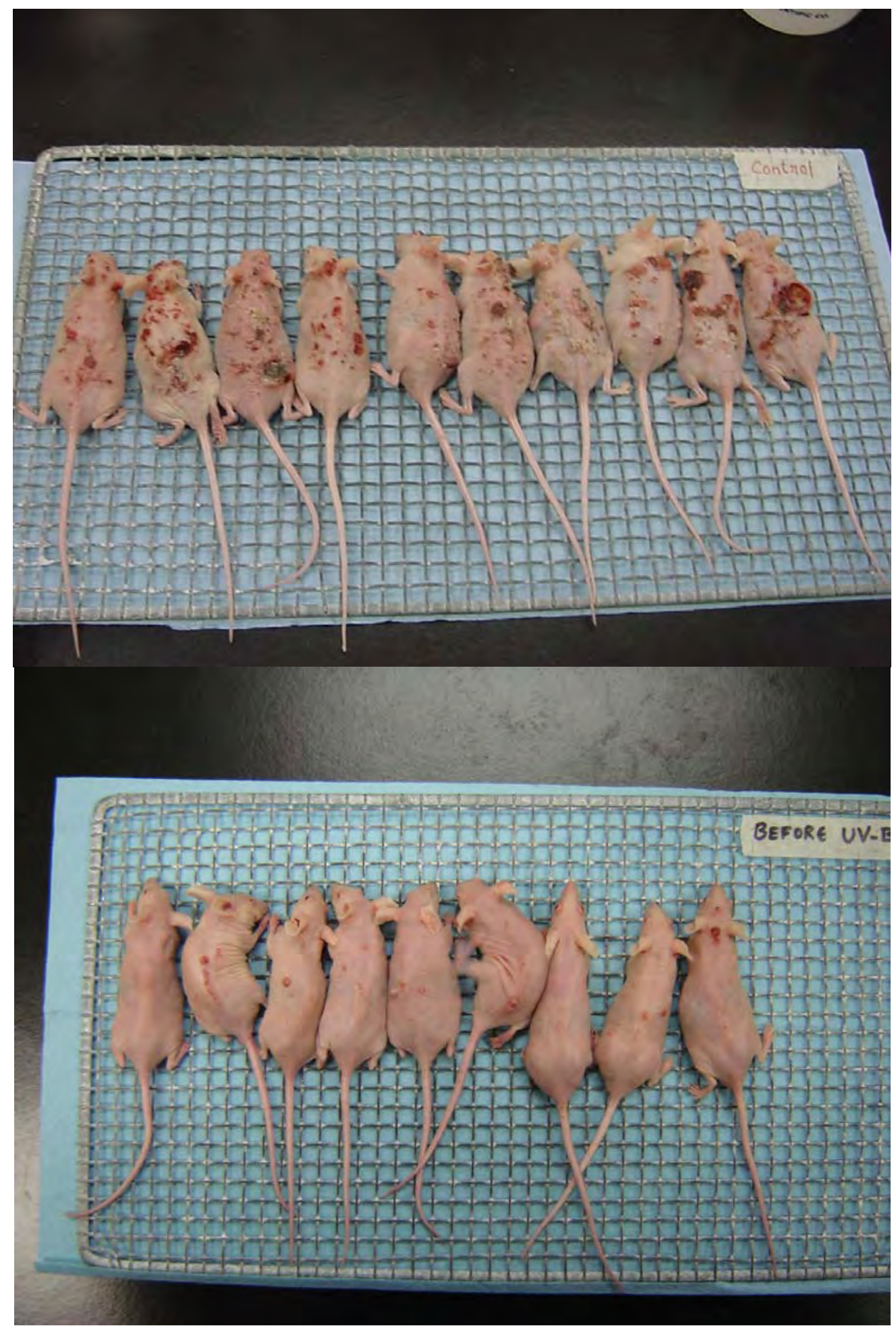


Based on the hypothesis that ST induces apoptosis by upregulating the caspase levels as its possible mechanism of chemoprevention against skin tumor development in CD-1 mice [16], the effects of ST on caspase-3 were analyzed. As shown in Figure 4, ST significantly $(P<0.05)$ upregulated the levels of caspase-3. We observed that the value of caspase-3 as compared in ST treated group was found to be 4.9 fold higher than Control group. In order to assess the mechanism of caspase-3 activation, a further study was carried out to find out whether caspase- 9 and -8 are activated as upstream effectors leading to caspase- 3 activation. We observed a significant $(P<0.05)$ increase in caspase- 9 and -8 activities as shown in Figure 5. The levels of caspase- 8 in ST treated group were found to be 5.7 fold higher than Control. Whereas the values of caspase-9 in ST treated group were 3.9 fold higher than Control. These results suggest that ST induced apoptotic cell death might be mediated by both extrinsic and intrinsic pathways of apoptosis in tumor cells in UVB-induced carcinogenesis.

Figure 4. The effects of ST pretreatment on proapoptotic proteins, caspase-3, 8 and 9, on UVB-irradiated skin tumors of SKH-1 hairless mice. Values represent mean \pm SE derived from at least three mice. Values of ST are percentages of control values quantitated by densitometry.

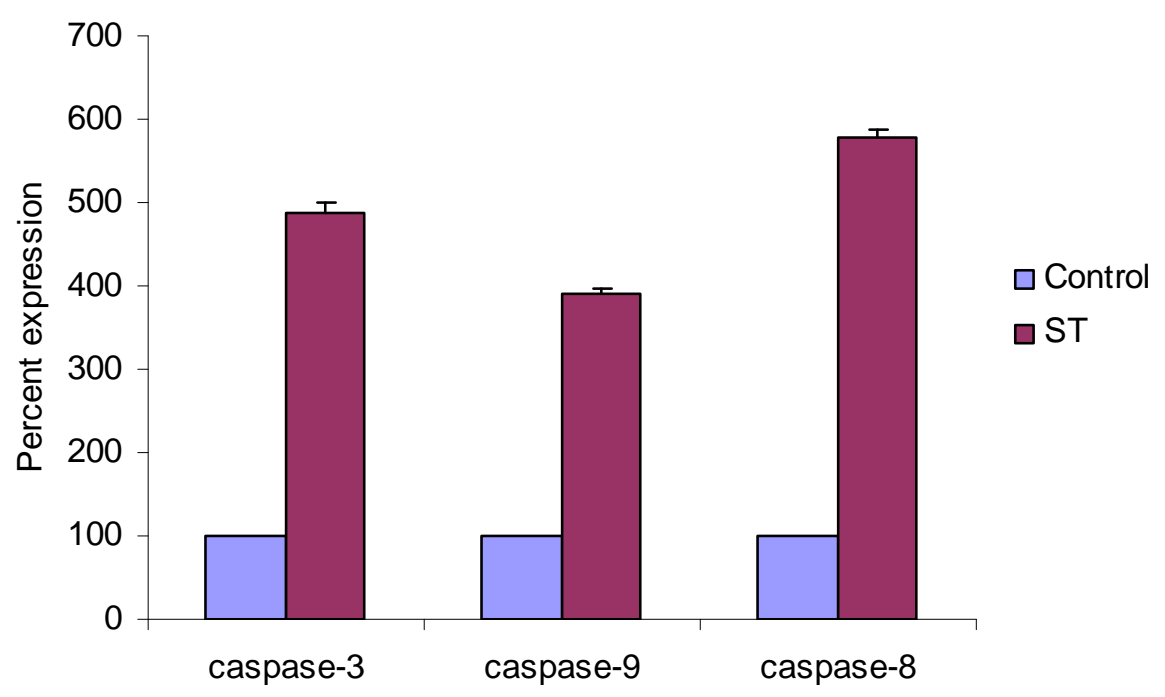

The gene p53 is one of the most frequently mutated in UVB-induced carcinogenesis [27]. It plays an important role in the induction of apoptosis through caspase dependent as well as caspase independent pathways [3]. The p53 tumor suppressor gene is essential in maintaining the genomic integrity of cells through its role in allowing repair of DNA damage or apoptosis [22]. A relationship between exogenous tumor induction and p53 expression has been reported in UVB-induced skin papillomas and carcinomas of the skin [28, 29]. The p53 tumor suppressor gene induces apoptosis in response to cellular stresses such as DNA damage, hypoxia, and oncogene activation [30]. In order to unravel the mechanism behind the induction of apoptosis by ST treatment we further assessed the levels of p53 in UVB-induced carcinogenesis. As expected there was a significant $(P<0.05)$ upregulation of p53 levels in ST treated group compared to Control. The value of p53 was found to be 1.9 fold higher in ST treated group when compared to the Control; data are shown in Figure 5. 
Figure 5. The effects of ST pretreatment on p53 on UVB-irradiated skin tumors of SKH1 hairless mice. Values represent mean \pm SE derived from at least three mice. Values of ST are percentages of control values quantitated by densitometry.

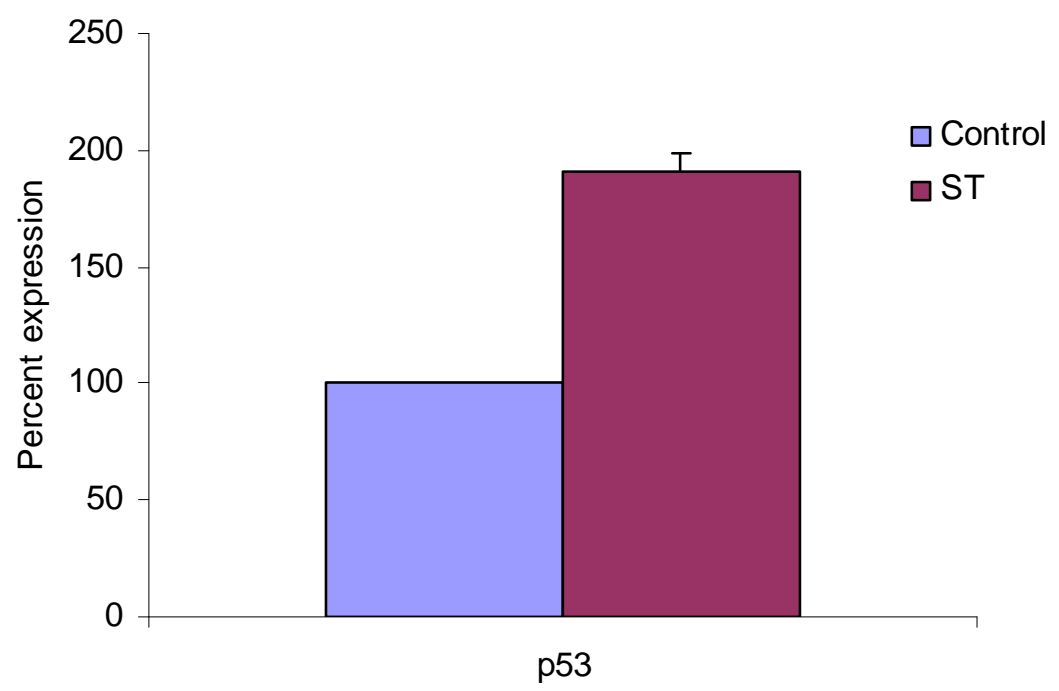

\section{Conclusions}

Taken together from the above results, the effectiveness of ST as chemopreventive agent appears to be very promising in UVB-induced skin cancer control. The mechanism behind its chemoprevention might be possibly by inducing apoptosis, upregulating p53, contributing to its overall cancer chemopreventive effects in the UVB-induced mouse skin cancer model.

\section{Experimental}

\subsection{General}

Sarcophine was isolated from the soft coral Sarcophyton glaucum collected from several locations of the Red Sea in Egypt. Phenylmethylsulfonylfluoride (PMSF), Tween 20, sodium chloride, ammonium persulphate, sodium lauryl sulphate, acetic acid, leupeptin and pepstatin were obtained from Sigma Chemical Company (St. Louis, MO). Acrylamide was purchased from Bio Rad Laboratories, (Hercules, CA), glycine, sodium dodecyl sulphate (SDS) and Tris from USB Corporation (Cleveland, $\mathrm{OH}$ ), and nitrocellulose membrane from Bioexpress (Kaysville, UT). Primary antibodies against caspase-3 were purchased from Cayman Chemical Company (Ann Arbor, MI), primary antibody against caspase-8, -9, and horseradish peroxidase conjugated goat anti-rabbit secondary antibodies were purchased from BD Biosciences (Rockville, MD) and p53 from Abcam Inc. (Cambridge, USA). ECL Kit was bought from Amersham Biosciences (Piscataway, NJ). Other reagents were obtained in their highest purity grade available commercially. 


\subsection{Materials}

Sarcophine was isolated from the soft coral Sarcophyton glaucum by multiple extraction with petroleum ether at room temperature following the reported procedure [19] at the laboratories of the Department of Pharmacognosy, Faculty of Pharmacy, Suez Canal University, Ismailia, Egypt. The dried extract was evaporated under reduced pressure and chromatographed on silica gel column using hexane: ethyl acetate (1:2) as eluent. Pure sarcophine was obtained by crystallization from ethanol. ST was synthesized according to the folowing procedure: Sarcophine was reduced to its lactone ringopened analogue (50 mg, $0.16 \mathrm{mmol}$ ) to which selenium dioxide (35.5 mg, $0.32 \mathrm{mmol}$ ) in dry 1,4dioxane $(15 \mathrm{~mL})$ was added and the reaction mixture was stirred at room temperature for $4 \mathrm{~h}$. Water was then added, and the product was extracted with $\mathrm{CH}_{2} \mathrm{Cl}_{2}$. Saturated $\mathrm{NaHCO}_{3}$ solution was used to wash the $\mathrm{CH}_{2} \mathrm{Cl}_{2}$ layer which was dried over anhydrous $\mathrm{Na}_{2} \mathrm{SO}_{4}$. The solvent was evaporated and the residue was chromatographed on silica gel using hexane - acetone (1:1) as an eluent to obtain ST (12 mg, 23\%) [18].

\subsection{UVB-induced Tumorigenesis Protocol:}

\subsubsection{UVB Source:}

The UVB irradiation unit, manufactured by Daavlin Corporation (Ohio, USA) consists of four UVB lamps. The exposure dose can be controlled by using two Daavlin flex control integrating dosimeters. The dose of UVB exposure is expressed in millijoules $/ \mathrm{cm}^{2}$.

\subsubsection{Animals:}

Female SKH-1 mice (5 weeks old) were purchased from Charles River Laboratories (Wilmington, MA). The animals were housed ten per cage at $22 \pm 1^{0} \mathrm{C}$ and $50 \pm 10 \%$ relative humidity and subjected to a $12 \mathrm{~h}$ light/ $12 \mathrm{~h}$ dark cycle in the College of Pharmacy animal facility. They were acclimatized for 1 week before use and provided food and water ad libitum.

\subsubsection{Experiment:}

The tumorigenesis protocol as described by Dwivedi et al. [31] was used. Female SKH-1 mice randomly divided into two groups, Control and ST treated. Both initiation as well as promotion was induced by UVB radiation $\left(180 \mathrm{~mJ} / \mathrm{cm}^{2}\right)$. During the initiation phase, Control was treated with acetone $(100 \mu \mathrm{L})$ and ST treated with ST (100 $\mu \mathrm{L}, 30 \mu \mathrm{g} / 100 \mu \mathrm{L}$ of acetone) one hour prior to UVB treatment. This was done every day and was continued until 14 days. Ten days after the initiation phase, promotion phase was started. During the promotion phase, both Control and ST treated groups were treated in the same way as they were treated during the initiation phase. But during the promotion phase, the treatment was done only twice a week (Tuesday and Friday) and was continued until the next 30 weeks. Tumor counts and group weights were taken once every week. 


\subsection{Lysate Preparation}

Mice from UVB-induced tumorigenesis protocol were sacrificed after 30 weeks by cervical dislocation and epidermis was collected from mice of Control and ST Treated groups respectively. The fat and tumors in the skin of these three groups were scraped by scalpel, and then epidermis was homogenized by an OMNI GLH-115 homogenizer in $0.1 \mathrm{mM}$ Tris-HCl (pH 7.4) containing $0.15 \mathrm{M}$ sodium chloride. The epidermal homogenate was filtered by cheesecloth and then filtrate was centrifuged at 10,000 g for $20 \mathrm{~min}$ in the Beckman J2-21 Centrifuge. This pellet combined with 5\% SDS containing 1\% $100 \mathrm{mM}$ PMSF, 0.5\% leupepth and 0.5\% pepstatin was allowed to pass through $25 \mathrm{G}$ needle and centrifuged at 13,000 g for $20 \mathrm{~min}$. The supernatant was collected, allowed to pass through $25 \mathrm{G}$ needle again and heated in heating block $\left(100{ }^{\circ} \mathrm{C}\right)$ for $5 \mathrm{~min}$. and epidermis was collected from mice of Control and ST treated groups respectively. The cell pellet of mice obtained as a result of ultracentrifugation is taken in an Eppendorf tube and lysed in 5\% SDS containing protease inhibitors leupeptin, pepstatin and phenyl methyl suphonyl floride (PMSF) as described by Kundoor et al. [16] and Zhang et al. [32].

\subsection{Western Blot Analysis of Caspase -3, -8, -9 and p53}

Protein concentration was measured in each cell lysate by the protein assay (Pierce, Illinois) with albumin as a standard. Equal amounts of protein lysates (60 $\mu \mathrm{g}$ ) were resolved on $12.5 \%$ SDS-polyacrylamide gels and transferred to nitrocellulose membrane. Membranes were blocked for $1 \mathrm{~h}$ in $5 \%$ skim milk in TBS (10 mM Tris, $100 \mathrm{mM} \mathrm{NaCl}$ ), and then probed with primary antibodies against caspase-3, -8, -9 and p53. The secondary antibodies conjugated to horseradish peroxidase were used for development with the enhanced chemiluminescence detection (ECL) kit. The Western blots were quantified using a UVP Biochem Gel Documentation system (UVP, Inc., Upland, California). Caspase $-3,8-,-9$ and p53 data were normalized using beta-actin as the housekeeping protein used routinely in our laboratories.

\subsection{Statistical Analysis}

The INSTAT software package (Graph Pad, San Diego, CA) was used to analyze the data. Chi Square was used for analyzing the data on tumor incidence. Student's t test was used to compare the tumor multiplicity, weight gain, caspase-3, -8, -9 and p53 levels in UVB-induced carcinogenesis. Significance in all the cases was considered at $P<0.05$.

\section{Acknowledgements}

This study was supported by Juhnke Funds from SDSU Foundation. 


\section{References}

1. Cancer Facts \& Figures-2007; American Cancer Society Publication; http://www.cancer.org

2. Mallikarjuna, D.G.S.; Singh, R.; Agarwal, C.; Agarwal, R. Silibinin protects against photocarcinogenesis via modulation of cell cycle regulators, mitogen-activated protein kinase, and Akt signaling. Cancer Res. 2004, 64, 6349-6356

3. Soehnge, H.; Ouhtit, A.; Ananthaswamy, O. N. Mechanisms of induction of skin cancer by UV radiation. Front. Biosci. 1997, 2, 538-551

4. Kraemer, K. Sunlight and skin cancer: another link revealed. Proc. Natl. Acad. Sci. 1997, 94, 1114

5. Agarwal, R.; Mukhtar, H. Cutaneous chemical carcinogens. In Pharmacology of the Skin. Mukhtar, H., ed.; CRC Press: Boca Raton, New York, 1991; pp. 371-387

6. Tornaletti, S.; Pfeifer, G.P. UV damage and repair mechanisms in mammalian cells Bioessays 1996, 18, 221-228

7. Pentland, A. P. Signal transduction mechanisms in photocarcinogenesis. Photochem. Photobiol. 1996, 63, 379-380

8. Melnikova, V. O.; Ananthaswamy, H. N. Cellular and molecular events leading to the development of skin cancer. Mutat. Res. 2005, 571, 91-106

9. Gu, M.; Singh, R. P.; Dhanalakshmi, S.; Agarwal, C.; Agarwal, R. Silibinin inhibits inflammatory and angiogenic attributes in photocarcinogenesis in SKH-1 hairless mice. Cancer Res. 2007, 67, 3483-3491

10. Greenleee, R. T; Hill-Harmon, M. B.; Murray, T.; Thun, M. Cancer statistics. CA Cancer J. Clin. 2001, 51, 15-36

11. Gupta, S.; Mukhtar, H. Chemoprevention of skin cancer: Current status and future prospects. Cancer Metast. Rev. 2002, 21, 363-380

12. Lamson, D. W.; Brignall, M.S. Natural agents in the prevention of cancer, part two: Preclinical data and chemoprevention for common cancers. Altern. Med. Rev. 2001, 6, 167-187

13. Ley, R. D.; Reeve, V. E. Chemoprevention of ultraviolet radiation-induced skin cancer. Environ. Health Perspect. 1997, 105, 981-984

14. Kelloff, G. J.; Sigman, C. C.; Greenwald, P. Cancer Chemoprevention: Progress and promise. Eur. J. Cancer. 1999, 35, 2031-2038

15. Bickers, D. R. and Athar, M. Novel approaches to chemoprevention of skin cancer. J. Dermatol. 2000, 27, 691-695

16. Haefner, B. Drugs from the deep: Marine natural products as drug candidates. Drug Discov. Today 2003, 8, 536-544

17. Fahmy, H.; Khalifa, S.; Konoshima, T.; Zjawiony, J. K An improved synthesis of 7,9-epoxy-1, 3,11-cembratriene-15 $\mathrm{R}(\alpha), 16$-diol, a cembranoid of marine origin with apotent cancer chemopreventive activity. Mar. Drugs 2004, 2, 1-7

18. Katsuyama, I.; Fahmy, H.; Zjawiony, J. K.; Khalifa, S.; Kilada, R. W.; Konoshima, T.; Takasaki, M.; Tokuda, H. Semisynthesis of new sarcophine derivatives with chemopreventive activity. $J$. Nat. Prod. 2002, 65, 1809-1814 
19. Ne'eman, A.; Fishelson, L.; Kashman, Y. Sarcophine-A new Toxin from the soft coral Sarcophyton glaucum (Alcyonaria). Toxicon 1974, 12, 593-598

20. Kundoor, V.; Zhang, X.; Khalifa, S.; Fahmy, H.; Dwivedi, C. A possible mechanism of action of the chemopreventive effects of sarcotriol on skin tumor development in CD-1 mice. Mar. Drugs 2006, 4, 274-285

21. Kaur, M.; Agarwal, C.; Singh, R. P.; Guan, X.; Dwivedi, C.; Agarwal, R. Skin cancer chemopreventive agent, alpha-santalol, induces apoptotic death of human epidermoid carcinoma A431 cells via caspase activation together with dissipation of mitochondrial membrane potential and cytochrome $c$ release. Carcinogenesis 2005, 26, 369-380

22. Sun, S. Y.; Jr. Hail N.; Lotan, R. Apoptosis as a novel target for cancer chemoprevention. J. Natl. Cancer Inst. 2004, 96, 662-672

23. Wattenberg, L. W. Chemoprevention of cancer by naturally occurring and synthetic compounds. In Cancer Prevention; Watterberg, L.; Lipkin, M.; Boone, C.; Kelloff, G., eds; CRC Press: Boca Raton, FL, 1992; pp. 19-39

24. Nicholson, D. W.; Thornberry, N. A. Caspase: Killer proteases. Trends Biochem. Sci. 1997, 22, 299-306

25. Ghobrial, I. M.; Witzig, T. E.; Adjei, A. A. Targeting apoptosis pathways in cancer therapy. CA Cancer J. Clin. 2005, 55, 178-194

26. Li, P.; Nijhawan, D.; Budihardjo, L.; Srinivasula, S. M.; Ahmad, M.; Alnemri, E. S.; Wang, X. Cytochrome c and dATP-dependent formation of Apaf-1/caspase-9 complex initiates an apoptotic protease cascade. Cell 1997, 91, 479-489

27. Stenback, F.; Makinen, M.; Jussila, T. p53 expression in skin carcinogenesis and its relationship to cell proliferation and tumor growth. Eur. J. Cancer 1998, 34, 1415 - 1424

28. Ruggeri, B.; Caamano, J. and Goodrow, T. Alterations of the p53 tumor suppressor gene during mouse skin tumor progression, Cancer Res., 1991, 51, 6615-6621

29. Ruggeri, B.; DiRado, M.; Zhang, S. Y.; Bauer, B.; Goodrow, T.; Kleinszanto, A. J. Benzo (a) pyrene-induced murine skin tumors exihibit frequent and characteristic $\mathrm{G}$ to $\mathrm{T}$ mutations in the p53 gene. Proc. Natl. Acad. Sci. 1993, 90, 1013-1017

30. Shen, Y.; White, E. p53-dependent apoptosis pathways. Adv. Cancer Res. 2001, 82, 55-84

31. Dwivedi, C.; Valluri, H. B.; Guan, X.; Agarwal, R. Chemopreventive effects of alpha-santalol on ultraviolet B radiation-induced skin tumor development in SKH-1 hairless mice. Carcinogenesis 2006, 27, 1917-1922

32. Zhang, X.; Kundoor, V.; Khalifa, S.; Zeman, D.; Fahmy, H.; Dwivedi, C. Chemopreventive Effects of Sarcophine-diol on Skin Tumor Development in CD-1 Mice. Cancer Lett. 2007, 253, 53-59

Sample Availability: Available from the authors.

(c) 2007 by MDPI (http://www.mdpi.org). Reproduction is permitted for noncommercial purposes. 